\title{
Guest Editorial: Advanced Technologies and Services for Multimedia Big Data Processing
}

\author{
Young-Sik Jeong ${ }^{1} \cdot$ Mei-Ling Shyu ${ }^{2} \cdot$ Guandong $\mathrm{Xu}^{3}$ • \\ Roland R. Wagner ${ }^{4}$
}

Published online: 30 April 2015

(C) Springer Science+Business Media New York 2015

This special issue focuses on advanced technologies and services for multimedia big data processing for future computing environments, including, but not limited to, heterogeneous data collection technologies, storage technologies, management technologies, processing technologies, analysis technologies, data mining, DBMS technology, intelligent technologies, information visualization technologies, convergence technologies, platform technologies, security, 3D visualization, distributed processing technologies, cloud service technologies, semantic of social media, service applications, new technologies for healthcare, new frontier platform, and services and technologies of manufacturing and education for multimedia big data processing. All papers focus on novel approaches for advanced technologies and services for multimedia big data processing and present high quality results for tackling problems arising from the ever-growing advanced technologies, services for multimedia big data processing and services for future computing environments.

In recent years, there has been a substantial amount of work on big data analytics using Hadoop-based platforms running on large clusters of computers $[1,2,4,5,7,9]$. In particular, multimedia and individuals with smartphones on social network sites will continue to fuel exponential growth. Big data-large pools of data that can be captured, communicated,

Young-Sik Jeong

ysjeong@dongguk.edu

Mei-Ling Shyu

shyu@miami.edu

Guandong Xu

guandong.xu@vu.edu.au

Roland R. Wagner

rwagner@faw.uni-linz.ac.at

1 Department of Multimedia Engineering, Dongguk University, Seoul, South Korea

2 Department of Electrical and Computer Engineering, University of Miami, Coral Gables, FL 33124, USA

3 Advanced Analytics Institute, University of Technology, Sydney, Australia

4 Institute for Applied Knowledge Processing (FAW), Johannes Kepler University Linz, Linz, Austria 
aggregated, stored, and analyzed - is now part of every sector and function of the global economy. "Big Data" refers to datasets whose size is beyond the ability of typical database software tools to capture, store, manage, and analyze. This definition is intentionally subjective and incorporates a moving definition of how big a dataset needs to be in order to be considered big data $[3,5,6,8]$.

This special issue aims to foster the dissemination of high quality research in methods, theories, techniques and tools concerning active multimedia big data technology in the coming era. The emerging applications and usages provide tailored, precise solutions wherever and whenever beings are active and extremely concentrated. Original research articles were solicited in all aspects of theoretical studies, practical applications, new communication technologies and experimental prototypes. This technology is being driven by and used in a wide range of academic, research, and commercial application areas.

We received many manuscripts, but only 11 papers of high quality were finally selected for this special issue. Each manuscript selected was blindly peer reviewed by at least three reviewers consisting of guest editors and external reviewers. The selected papers focus on novel approaches for advanced technologies and services for multimedia big data processing and present high quality results for tackling problems arising from the ever-growing advanced technologies and services for future computing. We present a brief overview of each paper below.

The first paper by Fan-Hsun Tseng, et al. proposed that social media services should be established with a well-defined infrastructure (10.1007/s11042-014-2086-z). The authors show that the VM migration problem in a cloud data center is formulated based on mixed integer linear programming, and the VM Allocation algorithm is proposed to construct a stable, robust, balanced network. Moreover, they specifically focus on the VM migration. The proposed algorithm not only achieves the lower number of VM migration and total cost, but also balances the utilization between PMs.

Fatos Xhafa et al. designed a secure cloud-based EHR system using ABE (10.1007/s11042013-1829-6). This system allows the patients to selectively share their PHRs with physicians by performing encryption under their current symptoms, but without knowing the precise description of their illnesses or the departments of physicians needed in medical treatment.

Wei Song and Kyungeun Cho proposed a real-time large-scale terrain modeling system for mobile robots (10.1007/s11042-013-1669-4). The proposed method was found to be effective in an outdoor environment for a mobile robot with a LiDAR sensor, video camera, GPS receiver, and gyroscope. They constructed a grid-based flag map to register the sensed 3D point clouds in the terrain model without redundancy. Subsequently, they applied a textured mesh and particle rendering method to represent the reconstructed terrain model.

Zezhong Zhang et al. proposed the first authentication scheme with user anonymity for the SIP using the ECC (10.1007/s11042-014-1885-6). Security analysis shows the proposed scheme not only is secure against common attacks such as replay attack, password guessing attack, man-in-the-middle attack, Denning-Sacco attack, stolen-verifier attack, but also provides user anonymity, mutual authentication, known-key security, session key security, and perfect forward secrecy.

Simon S. H. Park et al. developed a new measure to compute the distances effectively between multiple categorical data on which it incorporates the structural topology as well as the data distribution (10.1007/s11042-014-1914-5). The conventional approaches have not covered the multiple categorical structures, but the CTSM partly covered the issue. The CTSM, however, has exposed shortcomings so that the number of sibling nodes has not been considered and only depends on hop degrees. Their new measure, MCSM, has solved these two issues successfully 
and outperformed conventional approaches so that they can claim that MCSM is a solid and robust measure for multiple categorical data.

Wei-Jian $\mathrm{Xu}$ et al. proposed a reduced reference evaluation model named RR-PEVQ (Reduced Reference Perceptual Evaluation of Video Quality) for weighing the active video area (10.1007/s11042-014-1903-8). According to the experimental results, the RR-PEVQ evaluation score is similar to that of the full reference PEVQ and the proposed method's practicability was greatly improved for big data purposes.

Neil Y. Yen et al. discussed an emerging model for the understanding (and creation) of social empowered data and its derivative contexts (10.1007/s11042-014-1941-2). Furthermore, the management and reuse of such information are specifically concentrated [presented?]. Considering the dramatic growth of data, a theoretical design of the state machine to collect contexts, such as activity logs, behavior, etc., from end users was proposed. This framework is featured by its core approach on data processing as it highlighted the importance of potential fusion on data, algorithm, and results. Given a concrete usage scenario in social web, this state machine is expected to draw attention from users, keep them, and then predict their intentions through the analysis of collected contexts in the future. The framework was also implemented on an open platform that allows add-on services from third-party developers so that the user contexts can be collected comprehensively and objectively. Although there are still limitations and some work remains, it is certain that the construction and development of a system to provide smart business services is a worthwhile attempt to meet profound society needs.

Kuei-Fang Hsiao showed the need for a massive large-scale boost in two main dimensions of physical and mental health enhancement (10.1007/s11042-013-1649-8). In order to solve this problem, this paper proposed a new low-cost and innovative adoption of augmented reality (AR) functions through an agile deployment of mobilebased augmented reality (mAR) embedded in massively available intelligent smartphones. In their proposed method a set of downloadable ARenabled embedded learning and exercising programs, designed upon users' historical and habitual improvement data, would enable a collective sequence of required activities and be individually optimized. At the system design level, upon the individually recorded data in various databases, it selects and configures the most suitable set of downloadable programs - a combination of mental and physical activities. From their experiment it provided some statistical results for two distinct application areas of mAR: 'exercising-rehab' and 'lifelong learning'. Three sets of results showed the age related results for three user critical features of 'ease of use,' 'usefulness' and 'user attitude'. Further analysis of data through modeling helps to provide a systematic design procedure based on user's age in conjunction with other variables.

Minkyung Kim et al. developed a lifelog management system that allows roaming users carrying smartphones to effectively record huge amounts of their daily behaviors (10.1007/s11042-013-1671-x). The prototype personal lifelog management system collects acceleration data from sensors, extracts major data from the sensed raw data and then transfers only indexed major data into a smartphone to use storage capacity at a low cost and reduce network transfer cost. They also proposed a layered data logging system for effective personal lifelog management. One significant aspect of the lifelog management system is the approach of hierarchical process to choose the optimal usage configuration of hierarchical computing and storage resources.

Shingchern D. You and Wei-Hwa Chen considered the problem of computing representative LRDs based on the MPEG-7 audio signature descriptors so that the comparison 
time for music identification can be significantly reduced (10.1007/s11042-013-1670-y). Although they can reduce the number of MPEG-7 audio signature descriptors by altering the scaling ratio and the bandwidth per band, experimental results show that this approach has poor identification accuracy. Consequently, other reduction methods should be used. In this paper, they examine block-average, PCA, Hadamard, Harr, and CDF 9/7 methods for this purpose. For the PCA, Hadamard, Haar, and CDF 9/7 approaches, they also considered different partition methods to compute LRDs. The results showed that different matrix partition methods are required for different reduction approaches. Simulation results showed that block average, PCA, Hadamard, and Harr approaches yield comparable performance, but the CDF 9/7 approach performs relatively poorly. In addition to the accuracy, they also compared the arithmetic operations required for each approach. If not considering the computational complexity, except the CDF $9 / 7$ approach, the remaining four methods may be used to produce representative LRDs. If the computational burden is very important, then the block average method is the best choice.

Jeong-Hoon Lee et al. studied an in-vehicle sensor database management system (DBMS) (10.1007/s11042-013-1672-9). In the proposed approach, simply called in-vehicle DBMS approach, DBMS inside the ego-vehicle manages gathers and processes traffic and sensor data onboard such as signal data and multimedia data, including map and image data. They classify the requirements of applications using the in-vehicle DBMS into data modeling and query processing. They also proposed the system architecture for an in-vehicle DBMS which solves those issues and discussed database techniques offered by the system.

Our special thanks go to Dr. Borko Furht, Editor-in-Chief of Multimedia Tools and Applications, and the editorial staff for their valuable support throughout the preparation and publication of this special issue. We would like to thank all authors for their contributions to this special issue. We also extend our thanks to the external reviewers for their excellent help in reviewing the manuscripts.

\section{References}

1. Bughin J, Chui M, Manyika J (2010) Clouds, big data, and smart assets: ten tech-enabled business trends to watch. McKinsey Q 56(1):75-86

2. Buyya R, Broberg J, Goscinski AM (2010) Cloud computing: principles and paradigms. In: Zomaya AY (ed) Wiley Series on Parallel and Distributed Computing, vol 87. Wiley, Hoboken, p 664

3. Chen W-M, Lai C-J, Wang H-C, Chao H-C, Lo C-H (2011) H.264 video watermarking with secret image sharing. IET Image Process 5(4):349-354

4. Chou L-D, Chang Y-J, Yang J-Y, Peng W-S (2011) Knowledge management system for social network services. J Internet Technol 12(1):139-151

5. Gil J-M, Park JH, Jeong Y-S (2013) Data center selection based on neuro-fuzzy inference systems in cloud computing environments. J Supercomput 66(3):1194-1214

6. Hsiao KF, Rashvand HF (2011) Integrating body language movements in augmented reality learning environment. Human-Centric Comput Inf Sci (HCIS) 1(1):1-10

7. Jeong Y-S, Park JH (2013) High availability and efficient energy consumption for cloud computing service with grid infrastructure. Comput Electr Eng 39(1):15-23

8. Kim B, Youn C-H, Park Y-S, Lee Y, Choi W (2012) An adaptive workflow scheduling scheme based on an estimated data processing rate for next generation sequencing in cloud computing. J Inf Process Syst (JIPS) $8(4): 555-566$

9. Marinelli EE (2009) Hyrax: cloud computing on mobile devices using mapreduce. Technical Report, DTIC Document 


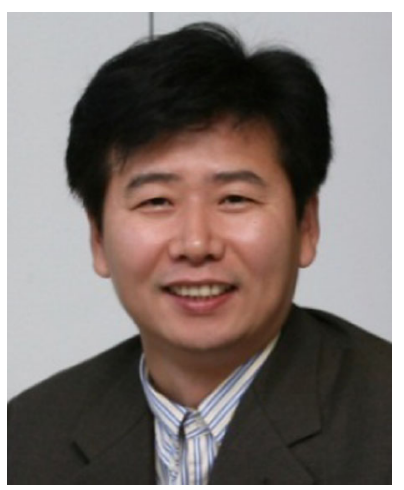

Young-Sik Jeong is a professor in the Department of Multimedia Engineering at Dongguk University in Korea. His research interests include big data technology, cloud computing, mobile computing, IoT (Internet of Things), and wireless sensor network applications. He received his B.S. degree in Mathematics and his M.S. and Ph.D. degrees in Computer Science and Engineering from Korea University in Seoul, Korea in 1987, 1989, and 1993, respectively. Since 1993, he has been serving as an IEC/TC 100 Korean Technical Committee member, as the IEC/TC 108 Chairman of Korean Technical Committee, and as an ISO/IEC JTC1 SC25 Korean Technical Committee member. He is an Editor-in-Chief of the Journal of Information Processing Systems, an associate editor of the International Journal of Communication Systems and an editor of Journal of Internet Technology, and an associate editor of the Journal of Human-Centric Computing. He is also is a member of the IEEE. http:// ucloud-lab.dongguk.edu

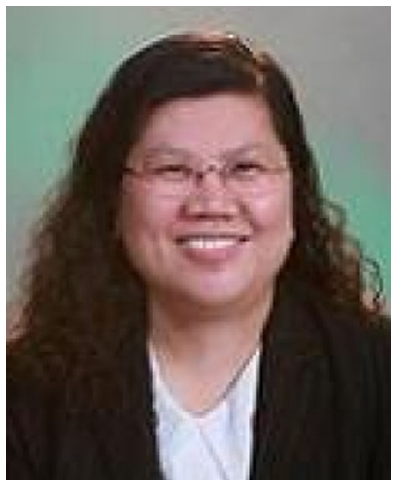

Dr. Mei-Ling Shyu is a full Professor at the Department of Electrical and Computer Engineering (ECE) at the University of Miami (UM) since June 2013. Prior to that, she was an Associate/Assistant Professor in ECE at UM since January 2000. She received her PhD degree from the School of Electrical and Computer Engineering and three Master degrees, all from Purdue University, West Lafayette, IN, USA. Her research interests include multimedia data mining, management and retrieval, and security. She has authored and co-authored more than 230 technical papers. Dr. Shyu was awarded the 2012 Computer Society Technical Achievement Award and ACM 2012 Distinguished Scientists Award. She received the Best Paper Awards from the IEEE International Symposium on Multimedia in 2013 and IEEE International Conference on Information Reuse and Integration in 2014 and 2012, Best Published Journal Article in IJMDEM for 2010 Award, Best Student Paper Award with her student from IEEE International Conference on Semantic Computing in 2009. She is a Fellow of SIRI. 


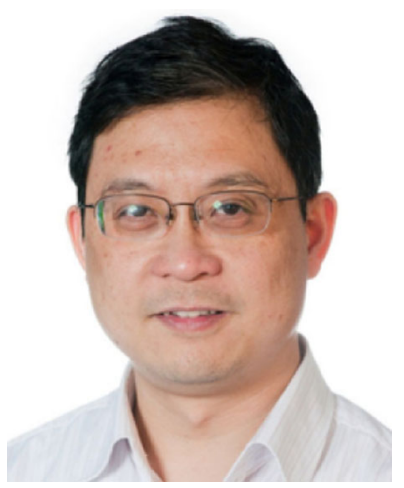

Dr. Guandong $\mathbf{X u}$ is a senior lecturer and Analytics Education Program Leader in the Advanced Analytics Institute, University of Technology Sydney and he received $\mathrm{PhD}$ degree in Computer Science from Victoria University, Australia in 2009. His research interests cover Web Data management and Web Analytics, Data Mining, Recommender Systems, Text Mining, Social Analytics, especially Social Network Analysis and Social Media Mining. In last 10 years, he has had over 90+ publications: He has published three authored monograph books with Springer and CRC press, one edited scientific book with IGI-global publisher, and seven conference proceedings along with dozens of journal and conference papers including CJ, WWWJ, KAIS, CCPE, KBS, ESWA, JIIS, IJCAI, AAAI, WWW, ICDM, CIKM, DASFAA, WISE, and PAKDD. He has been serving in editorial board or as guest editors for several international journals, such as the Computer Journal, Journal of Systems and Software and World Wide Web Journal, and he is the assistant Editor-in-Chief of World Wide Web Journal. He is also active in organizing or serving for international conferences and workshops. He is a PC CoChair of 2014 IEEE/ACM ASONAM conference. He holds IEEE and ACM membership.

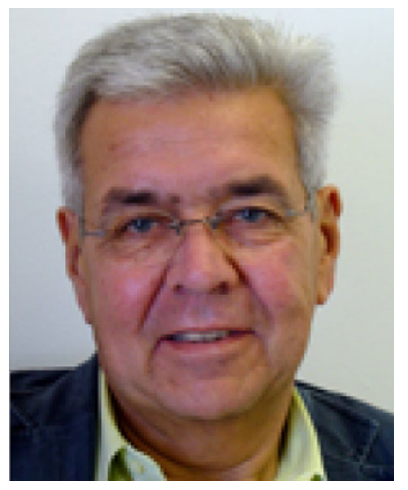

Roland Wagner is a Professor for Information Systems at the Johannes Kepler University of Linz. He is director of the Research Institute of Applied Knowledge Processing (FAW) and director of the Institute Integrated Studies ("integriert studieren"). He is managing various industrial and research projects in the areas of information systems, knowledge-based systems, electronic commerce and eAccessibility. He is teaching in the area of Information Systems and his current research interests are new database developments, object oriented database environment, data mining, data warehouses and eAccessibilty. 